\title{
A tablet-based memory enhancement application for older users: design approach
}

\author{
Lua Perimal-Lewis \\ Flinders University, South \\ Australia \\ lua.perimal- \\ lewis@flinders.edu.au
}

\author{
Anthony Maeder \\ Flinders University, South \\ Australia \\ anthony.maeder@flinders.edu.a \\ u \\ Jennifer Tieman \\ Flinders University, South Australia \\ jennifer.tieman@flinders.edu.au
}

\author{
Susan Gordon \\ Flinders University, South \\ Australia \\ sue.gordon@flinders.edu.au
}

\begin{abstract}
This paper provides a case study of the design process undertaken in producing a mobile tablet memory assistant solution which was intended for older adults (>65yo) living with early stage memory loss. We adopted an overall design framework consistent with "living laboratory" methodology, for which the associated design principles are: cocreation, multi-stakeholder participation, active user involvement, real-life setting, and multi-method approach. We describe here the detailed steps and provide examples of the application design decisions and outcomes, through successive stages of its evolution. Results of the various user engagements which informed our design choices and for validation of the artefact are presented.
\end{abstract}

\section{Introduction}

The proliferation of software applications in mobile consumer health settings raises questions of good practice in how to design and develop them to maximise acceptance, utility and benefits to the user. Often applications are developed independently of users and then sent to trial, only to discover design flaws through assumptions about user characteristics. This is especially disadvantageous when the application is intended for a user group which has specific limitations or expectations which will be imposed on the technology.

The case study presented in this paper addresses one such user group which provides a potential "new market" for this type of application, namely the ageing community. Senior citizens today were born in the postwar baby boomer era, and sociologically typically have high demands of personalized services and are technologically fairly competent. On the other hand, they experience many of the conventional losses of physical characteristics associated with ageing, such as sensory acuity and manual dexterity. In designing assistive mobile applications broadly for this group, it is necessary to address both these heightened expectations and to cater for their limitations.

The target application described here is a mobile tablet memory assistant solution which was intended for older adults (>65yo) experiencing early stage memory loss. As we age, short term memory loss tends to develop in most people, providing a source of frustration in everyday living and adversely affecting functional capacity and ability to manage some aspects of daily living. By designing a software assistant to overcome the simplest and commonest elements of memory failure, and some simple memory improvement activities for users, we aspired to offer a useful and usable tool to be integrated into their everyday life.

The application was aimed specifically as a consumer-centred self-care intervention for independent living older adults who are "ageing in place" and have self-identified as experiencing early stage memory loss. It was not expected that it would be subject to clinical or therapeutic use, nor part of a specific healthcare service component. The lack of such assistance interventions in automated form creates a health disparity for those who begin to experience early stage memory loss while living independently. They currently require human assistance to address their deficiency, which is infeasible to provide as it would require constant presence of a human assistant. In contrast, more effort has been made to provide interventions based on various technological aids to 
address disparities for those in a more advanced state of cognitive decline, as it is assumed these aids will be provided and supported through clinical channels.

Methodologies of user-centred and participatory design have been strongly advocated for development of health applications [1] and especially in socially diverse settings [2]. Our approach to designing this application followed a highly iterative and widely consultative approach to participatory design, using a set of so-called "living laboratory" principles [3]. Living laboratory methodology is seen as more radical than participatory design since engagement with older adults starts at the earliest point in the design process, where the older adults are given control of generating requirements and have opportunities to make design decisions and suggestions [4].

Historically, older adults are hesitant to engage with technology and so we sought to consider the research question of the effectiveness of choosing a methodology where the end users are at the centre of innovation. Creating an artefact 'with older adults' rather than 'for older adults' should empower these users to be more likely early adopters of the new artefact. Our paper reports on the steps followed and decisions made during that process, and the subsequent validation of the software in a pilot deployment.

\section{Methodology}

Age-related memory loss is a well-known effect based on deterioration in neurological pathways and brain matter [5]. Provision of a range of technologybased measures have been suggested [6] targeting common support needs such as scheduling and reminders for daily activities, recall of names and contact details, and access to communication and information sources. A scoping review of automated assistive solutions noted the value of providing multimodal functions in one system, while at the same time ensuring that the solution was controllable by the user in supporting desired activities rather than performing them independently of the user [7]. This approach is in harmony with the hypothesis that cognitive activity including purposeful cognitive tasks and structured cognitive exercising can slow or even arrest memory decline [8]. It is also understood that physical exercise has beneficial effects on age-related memory decline [9].

We therefore sought to develop a simple computer-based memory assistant solution which was intended for independently living older adults (>65yo) suffering from early stage memory loss, but as yet not clinically diagnosed with dementia or Mild Cognitive Impairment (MCI). For this reason, clinically validated screening tools were used to determine that the inclusion criteria were satisfied for our research participants.

From our review of the literature, we established that the functionality should include daily-context (e.g. time, place, weather, news), timed-event (e.g. calendar, daily schedule, alerts) and personal-communication (e.g. names, faces, phone) functions. We also accepted that the acknowledged value of functions enabling users to undertake cognitive training and physical activity, would justify their inclusion. Our project objective was therefore to design a solution including these components, which would be suitable for and adopted by older adults.

We recognized that the solution would need to be mobile due to the degree of acceptance emanating from the portability of such devices, with a need for a larger form factor display and interaction surface than a smartphone, to allow ease of use [10]. We selected a tablet as the physical platform on the basis of a recent study which indicated a preference for this type of computer technology access amongst older adults [11]. We desired a solution that was selfstanding rather than reliant on network communication and interaction with a host system, so that its functionality would not be compromised by related complexities of access control and connection.

We adopted an overall design framework consistent with "living laboratory" methodology [12]. This is a particular type of co-design process which relies on applying numerous highly iterative cycles in the design evolution pathway, involving a very broad range of different stakeholders who provide often contrasting views which must be resolved in the final form. The key associated principles of "living laboratory" methods are [13]:

1. Co-creation, deriving new ideas and interpretations jointly across several coordinated parties.

2. Multi-stakeholder participation, with democratising of options and decisions during the overall design.

3. Active user involvement, engaging the targeted end user group closely throughout the creative process.

4. Real-life setting, evolving and testing the product within the actual type of site in which it is intended to be used.

5. Multi-method approach, combining objective and subjective mechanisms for distilling design inputs and reaching choices.

Living laboratory methods encourage the inclusion of diverse user contributions for a well- 
rounded outcome [14]. Taking this into consideration, we then identified the following groups of stakeholders for inclusion in our design deliberations:

1. Project team members: university staff chief investigators in the research project.

2. External stakeholders: health care sector who were supporting the research along with project team members.

3. Business development stakeholders: business sector agents interested to develop the project beyond research offering.

4. Marketing/distribution stakeholders: government and business sector parties interested to promote adoption.

5. User community representatives: caregivers of people living with dementia, clinicians/geriatricians, local government community workers and managers, community-dwelling older adults.

6. Solution domain experts: independent university staff with experience in apps for older adults and technical staff with experience in ICT development.

7. Actual end-user population: Older adults aged $>65$ yo assessed according to approved research eligibility criteria to fit characteristics of early stage memory loss.

The project commenced with populating of a tabular scheme for consultation of each stakeholder group at each stage in the design sequence, and the type of consultation involved. Table 1 below outlines the "living laboratory" principles and their corresponding activities. In the next section, we present the results of user engagement which contributed to significant changes to the project.

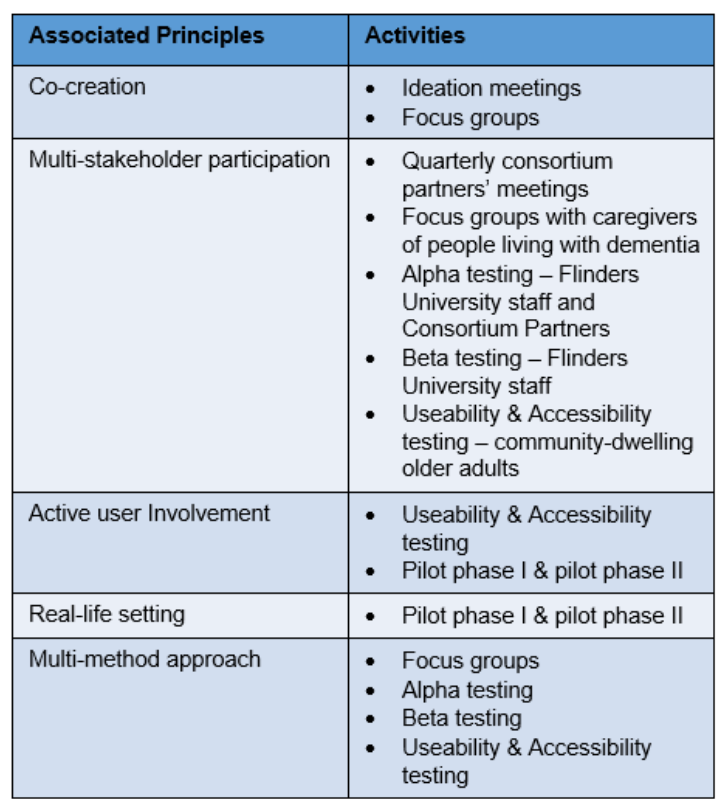

Table 1: Living laboratory methodology and associated activities

\section{Results}

In the sequence of design tasks for the project, we first identified a set of use cases and desirable usage characteristics for those structural elements of the solution aligned with the above defined functionality needs. Some loose constraints on the selection and disposition of the elements were sought from potential users and from expert consultation, to provide a high level description for the application. The result of this design stage was a conceptual model indicating the core functional components needed in the solution, expressed from a theoretical user perspective.

We then conducted focus groups with caregivers of people living with dementia $(n=6)$ to guide cocreation of the proposed solution further. Focus groups were deemed to be an appropriate method to use in this context, because the purpose of user involvement at this stage of the project was to gather feedback from the participants on the proposed solution which had predefined deliverables.

The first focus group considered a mock-up of the application which was done by us, complying to accessibility and usability aspects of $\mathrm{W} 3 \mathrm{C}$ guidelines [15]. All the functionalities of the proposed application were included, and each participant was given the opportunity to give feedback from the perspective of the person living with dementia and how they would respond if presented with such an application.

The second focus group was conducted two weeks later with the same group of caregivers. Another 
mock-up of the application was produced with refinements based on the feedback received from the first focus group. The positive feedback meant there was no need for another focus group. Figure 1 shows the changes in the front page of the application mock up, as a result of these focus group inputs.
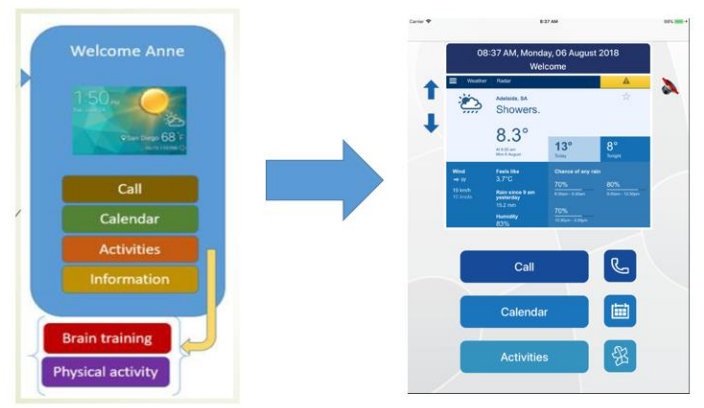

Figure 1: Transformation of first mock-up to final mock-up by focus group consultations.

The subsequent implementation task for the application was conducted through successive software creation and modification phases, inserting the different modules incrementally. These were refined by incorporating user feedback from alpha testing, followed by feedback from beta testing of the improved prototypes. The purpose of alpha and beta testing to was to test the application for any potential bugs and to establish features which were not consistent with optimal usability. Both these testing tasks were completed by research staff $(n=5)$ with experience as described in the previous section. This was then followed by user acceptance testing involving the project business partners $(n=2)$. The details of these tasks are summarized in Table 2 below.

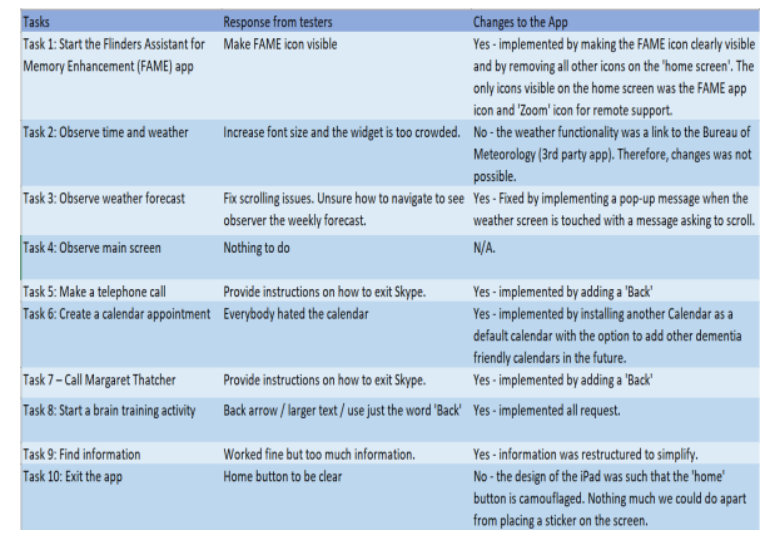

Table 2: Results from alpha testing and usability testing
Examples of the final contacts list and brain training activities screens derived in this implementation and testing phase are shown in Figure 2.

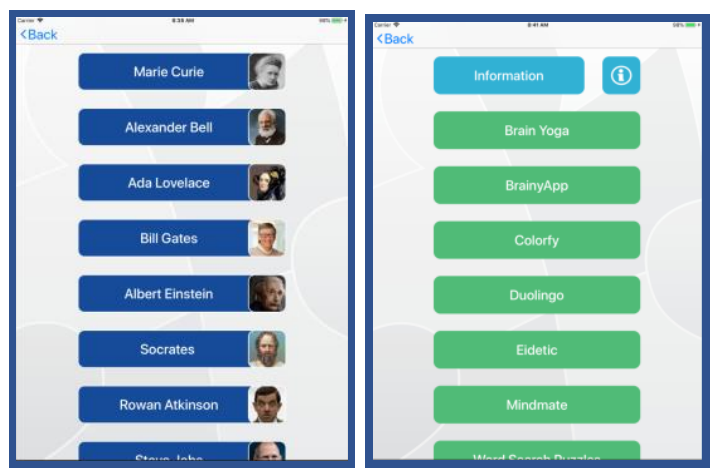

Figure 2: Refinement of screens from alpha and beta testing.

The following are comments from the testers of beta testing which validated the changes made to the app as a consequence of alpha testing:

- $\quad$ Easy to find the app on the tablet screen.

- Font is big enough and functionalities being demonstrated are clear.

- The app is very intuitive and I have not found serious problems.

- The app is intuitive and simple enough.

- I could not crash/freeze the app.

- Visual design of app is good - very simple to use, buttons are large $\&$ and screen is not overcrowded by graphics.

- The images accompanying the function buttons are self-explanatory.

- I enjoyed the ability to turn on and off the voice assistance.

- The buttons are very user friendly and bigger in size, suitable for the elderly population.

Simultaneously with the implementation phase, we engaged a local government council to recruit community-dwelling older adults $(n=5)$ aged $>65$ yo with no required experience in using smart phones or mobile devices, to conduct usability and accessibility testing. The purpose of this testing was to gauge whether the functional interface of the application was intuitive enough for this population. Each participant was allocated a moderator and an observer. The moderator was given a script to introduce the project and help with answering any questions the participants may have when undertaking the testing following predefined tasks related to using the different 
functionalities within the application. The observer documented how the participant performed each task and noted time taken to complete each. The tasks given were similar to the tasks listed in Table 2 but this time with outline of navigation steps required to complete the tasks.

All our participants were able to successfully complete the given tasks. They also enjoyed interacting with the app. The feedback was very similar to the results of alpha testing. This feedback was reassuring and the application was improved based on the observations made from the usability and accessibility testing (as shown in Table 2) before it was deemed ready to be tested in a real-life setting by the target end users in two separate pilots.

Participants were placed in the pilot phases for 12 weeks for each phase. Each screened eligible participant was loaned a study iPad with the application pre-loaded and customised with personal contacts data for that individual. Pilot phase I $(n=60)$ included brain training as an intervention and all other functionalities for participants to use. Pilot phase II $(n=60)$ included physical activity in addition to all phase I functionalities, with some minor improvements identified as a result of user experiences reported from phase I.

At the conclusion of the phases, participants were interviewed to determine their experiences and recommendations from the testing, and details of their usage of the application over the testing period were analysed. Completion of pilot phase II data is currently underway. Preliminary analysis of pilot phase I shows the following:

- $83 \%$ of participants answered that weather should be included in the app.

- $90 \%$ of the participants answered that call functionality should be included in the app.

- $79 \%$ of the participants answered that calendar should be included in the app.

- $100 \%$ of the participants answered that brain training should be included in the app.

- $79 \%$ of the participants enjoyed the brain training activities.

A majority of participants who favoured the weather functionality indicated that they use weather regularly as it helps with planning activities such as washing and outing. The participants who favored the call functionality liked it because of its simplicity and that they did not have to scroll through a long list of names. Participants who favoured the calendar functionality liked it because of the reminder/alert capabilities.
Overall the participants who did not think the weather or call functionalities should be included did not see the value of these, mainly because they already accessed weather information using other mediums and were already using call features on their phones. Those who thought the calendar functionality should be included had some experience with electronic calendars, and those who did not had existing habit of using a pen and paper calendar.

In addition to the extensive stakeholder involvement in the development of the application, we also undertook a post pilot user experience survey $(n=9)$. The results of the survey are shown in Table 3 below. The user experience survey validated and reinforced some of the design choices and also highlighted room for improvement, indicative of the value of the iterative process needed when applying "living laboratory" methods.

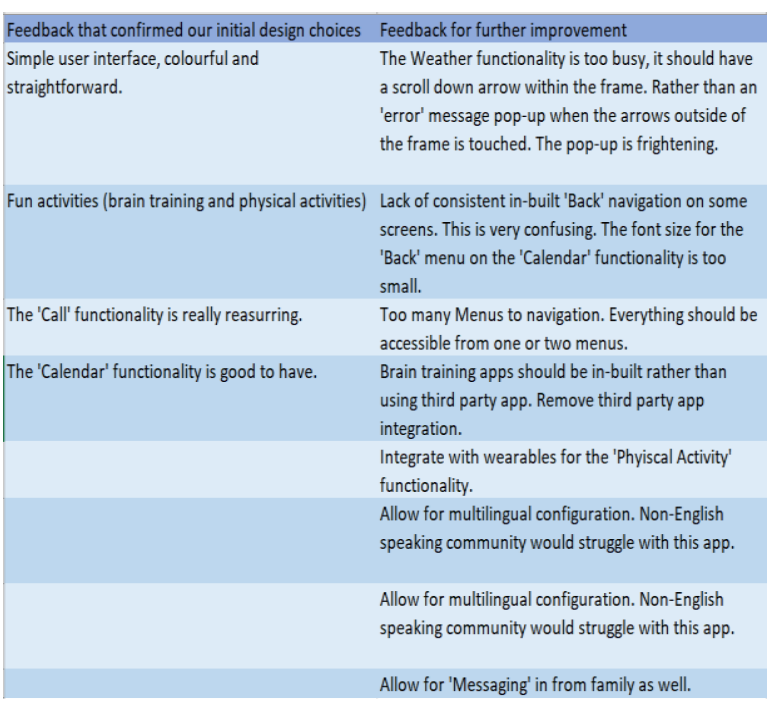

Table 3: Results from user experience survey

\section{Conclusion}

When designing solution for older adults who may have some limitations affecting their response in technology adoption, conventional requirement gathering followed by isolated software development for implementation may be a sub-optimal solution. This case study has demonstrated the value of employing "living laboratory" methods because the inputs of different stakeholders at various stages influenced the rollout of the project in different ways. While the development time was not shortened (taking 6 months from initial conceptualizing to final release version for Phase I testing) the quality of information received enabled design changes to be made on a 
consistent improvement and convergence trajectory, through active requirements gathering and implementation refinement. Successful solution design must be sympathetic to the deployment environment and the stakeholder ecosystem. The "living laboratory" methodology we adopted provided a mechanism to achieve this, with active user involvement in every stage of the development process.

\section{Acknowledgements}

This research was undertaken with financial support from the Australian Department of Health, through the Dementia and Aged Care Service Grant Scheme. In-kind support received from Dementia Australia and Southern Cross Care is also gratefully acknowledged.

\section{References}

[1] A.M. Kanstrup, A. Bygholm, P. Bertelsen \& C. Nohr eds. (2017). Participatory Design \& Health Information Technology, IOS Press, Amsterdam.

[2] E. Byrne \& S. Sahay (2007). Participatory design for social development: A South African case study on community-based health information systems. Information Technology for Development, 13(1), 7194.

[3] C. Dell'Era \& P. Landoni (2014). Living Lab: A methodology between user-centred design and participatory design. Creativity and Innovation Management, 23(2), 137-154.

[4] B. Bergvall-Kåreborn, D. Howcroft, A. Ståhlbröst \& A.M. Wikman (2010). Participation in Living Lab: Designing Systems with Users. IFIP Working Conference on Human Benefit through the Diffusion of Information Systems Design Science Research. Springer, Berlin, Heidelberg, 317-326.
[5] Small, G. W. (2002). What we need to know about age related memory loss. BMJ, 324(7352), 1502-1505.

[6] N. Kapur, E.L. Glisky, \& B.A. Wilson (2004). Technological memory aids for people with memory deficits. Neuropsychological Rehabilitation, 14(1-2), 41-60.

[7] N. Caprani, J. Greaney, \& N. Porter (2006). A review of memory aid devices for an ageing population. Psychology Journal, 4(3), 205-243.

[8] A. La Rue (2010). Healthy brain aging: role of cognitive reserve, cognitive stimulation, and cognitive exercises. Clinics in Geriatric Medicine, 26(1), 99-111.

[9] I. Bherer, K.I. Erickson \& T. Liu-Ambrose (2013). A review of the effects of physical activity and exercise on cognitive and brain functions in older adults. Journal of Aging Research, 2013.

[10] P. Joddrel \& A.J. Astell (2016). Studies involving people with dementia and touchscreen technology: a literature review. JMIR Rehabilitation and Assistive Technologies, 3(2), e10.

[11] D. Dasgupta, B. Chaudhry, E. Koh \& N.V. Chawla (2016). A survey of tablet applications for promoting successful aging in older adults. IEEE Access, 4, 9005-9017.

[12] The Living Lab Methodology Handbook, Lulea University of Technology (2013).

[13] P.S. Evans, A. Ståhlbröst \& K. Vervoort (2017). User engagement for large scale pilots in the Internet of Things. European Union: U4IoT Consortium.

[14] D. Schuurman, L. Coorevits, S. Logghe, K. Vandenbroucke, A. Georges \& B. Baccarne (2015). Co-creation in living labs: exploring the role of user characteristics on innovation contribution. International Journal of Services Sciences, 5(3-4), 199-219.

[15] Web Content Accessibility Guidelines (WCAG) 2.0, World Wide Web Consortium (2008). 